\title{
Guest editorial: a special issue on optimization techniques for business intelligence systems
}

\author{
Jie Lu · Guangquan Zhang
}

Received: 13 August 2010 / Accepted: 18 August 2010 / Published online: 29 August 2010

(C) Springer Science+Business Media, LLC. 2010

Business Intelligence refers to computer-based technologies, methodologies, tools, and systems for collecting, integrating, analyzing, and presenting large volumes of information to enable better business decision making. Increasingly, as businesses become more automated, data-driven, and real-time, business intelligence architecture evolves to support both strategic and operational levels of business decision making, which requires more advanced techniques and technical support. Aiming at better supporting business decision-making, business intelligence system is naturally a decision support system (DSS) in practice.

Among many core techniques of DSS, an optimization-based DSS whose various optimization methodologies and techniques are applied, aims to arrive at the best or most satisfactory decision out of a multitude of possible alternatives for business decision problems. Decision making has become more complicated and difficult in the current rapidly changing business environment. In recent years, both typical decision optimization such as multi-criteria decision making, and new decision intelligence techniques such as particle swarm optimization, have had unimaginable improvements. Particularly, through integrated with knowledge engineering and computational intelligence approaches, hybrid methodologies have been developed to deal with complex, uncertain, and un-structured decision optimization problems in businesses.

This Special Issue provides an updated overview of the research field in line with optimization techniques for business intelligence systems. It includes ten papers to present recent developments in methodologies, techniques and applications in optimization techniques for business intelligence systems from multiple aspects. The Special Issue covers three sub-topics: Intelligent multi-criteria optimization systems (papers 1-3); Optimization methodologies for business intelligence applications (papers 4-7); and Hybrid optimization algorithms by integration with computational intelligence and machine learning techniques (papers 8-10).

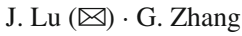

University of Technology, Sydney, P.O. Box 123, Broadway, NSW 2007, Australia

e-mail: jielu@it.uts.edu.au

G. Zhang

e-mail: zhangg@it.uts.edu.au 
The first paper by B. Vitoriano et al. (Spain) identifies a set of criteria and establishes a multi-criteria optimization model for humanitarian aid distribution.This model is the core of a DSS currently under development to assist organizations in charge of the distribution of humanitarian aid. The proposed criteria and the multi-criteria optimization model are futher examined by an illustrative case study based on the 2010 Haiticatastrophic earthquake, demonstrating the usefulness of the techniques.

The second paper by O. Kabak and D. Ruan (Belgium) presents a comparison study of various fuzzy multi-attribute decision making (MADM) methods in nuclear safeguards evaluation. After having investigated the applicability of four basic methods, namely, a SAW method, a TOPSIS approach, a linguistic approach, and a non-compensatory method for nuclear safeguards evaluation, the authors have proposed a new fuzzy MADM methodology by means of a cumulated-believe-degree approach for a better nuclear safeguards evaluation.

The third paper by X. Deng et al. (France) develops an intelligent multi-criteria DSS for quick, market-oriented textile material design. The authors have explored how a suitable online design of experiments can help product designers recursively finding the most suitable values for design factors with a very small number of trials. They first defined the degree of acceptability for a product prototype, then conducted modelling of the relationship between the process settings and the overall degree of acceptability. Based on this information, the authors have proposed a reinforcement learning-based method to generate the relevant operation setting space. Finally, the proposed system is evaluated by examining a case study of resilient-acoustic material design.

The fourth paper by Y. Gao et al. (Australia) proposes a Particle Swarm Optimization (PSO) algorithm for bi-level pricing problems in supply chains. It establishes two bi-level pricing models for the buyer and the vendor in a supply chain by designating the buyer and the vendor as the leader, respectively. To solve the problems defined by the two bi-level pricing models, a PSO based bi-level optimization algorithm is developed. A set of experiments illustrates that this PSO based bi-level optimization algorithm can achieve a profit increase for buyers or vendors if they are treated as the leaders under certain situations, compared to existing methods. The proposed PSO based bi-level optimization algorithm can also be used in other decision situations, such as power markets and transportation managements.

The fifth paper by D. Liu et al. (China) presents a new optimization approach including an applicable algorithm to learn interesting knowledge in dynamic business intelligence systems. An illustrated example is provided, with a comparison of the simulation results of the proposed algorithm with other methods to validate the proposed optimization approach.

The sixth paper by X. Yu et al. (Australia) proposes an improved training algorithm for neural networks learning based on a fast terminal attractor concept. It demonstrates that the new algorithm enables a fast convergence, both at a distance from, and in a close range to, the ideal weights. A condition to guarantee avoidance of the singularity problem is derived. Simulation studies are presented to show the effective training performance of the new algorithm in comparison with typical existing algorithms with an application for stock market index prediction.

The seventh paper by M. Raza et al. (Australia) develops a set of Maturity, Distance and Density (MD2) metrics for optimizing trust prediction for business intelligence in a web-based environment. The authors presented formal definitions of trust modelling, trust prediction, trust determination and confidence levels. They also discussed a case study to demonstrate how the proposed model would result in an optimized trust prediction.

The eighth paper by J. Pangilinan and G. Janssens (Philippines and Belgium) investigates the performance of evolutionary algorithms in the optimization aspects of oblique decision tree construction, and describes their performance with respect to classification accuracy, tree 
size, and Pareto-optimality of their solution sets. The performance of the evolutionary algorithms is analyzed and compared with the performance of exhaustive (traditional) decision tree classifiers on several benchmark data-sets. The results demonstrate that the classification accuracy and tree sizes generated by evolutionary algorithms are comparable with those generated by traditional methods in all the sample data-sets.

The ninth paper by Q. Zhou et al. (China) discusses construction of composite search directions with parameters in quadratic interpolation models. The authors first introduced weighted composite search directions to develop the quadratic approximation methods. A set of composite directions is obtained, based on the properties of simplex methods, and is used to construct trust region sub-problems. These sub-problems are then solved with an algorithm which finds solutions for benchmark optimization problems. The computation results show that, compared with other tested problems, the improved quadratic approximation methods significantly reduce the number of function evaluations, for most tested problems. Potential applications for this improved quadratic interpolation method in business intelligence systems is also discussed.

The last paper by J. Liu et al. (UK) proposes a new optimization algorithm for learning consistent belief rule-base from examples. The paper focuses on the consistency of belief rule base (BRB), given that the consistency conditions are often violated if the system is generated from real world data. The measurement of inconsistency of BRB is given and is incorporated in the objective function of the optimization algorithm. This process is formulated as a nonlinear constraint optimization problem. A numerical example is provided to demonstrate the effectiveness of the proposed algorithm.

We wish to thank Prof. Panos M. Pardalos, Editor-in-Chief of the Journal of Global Optimization for his encouragement, support and guidance to the guest editors during the preparation of this Special Issue. We also thank all the contributors and referees for their time and assistance with the Special Issue. 